\title{
Report of the Working Group on the Composition of Ultra-High Energy Cosmic Rays
}

\author{
Michael Unger ${ }^{* a, b}$, for the Pierre Auger Collaboration ${ }^{c}$ and the Telescope Array \\ Collaboration $^{d}$ \\ ${ }^{a}$ Center for Cosmology and Particle Physics, New York University, 4 Washington Place, New \\ York, NY 10003, USA \\ ${ }^{b}$ Institut für Kernphysik, Karlsruher Institut für Technologie, Postfach 3640, 76021 Karlsruhe, \\ Germany \\ ${ }^{c}$ Observatorio Pierre Auger, Av. San Martín Norte 304, 5613 Malargüe, Argentina \\ E-mail: auger_spokespersons@fnal.gov \\ Full author list: http://www.auger.org/archive/authors_2015_ICRC.html \\ ${ }^{d}$ Telescope Array Project 201 James Fletcher Bldg. 115 S. 1400 Salt Lake City, UT 84112-0830 \\ E-mail: ta-icrc@cosmic.utah.edu \\ Full author list: http://www.telescopearray.org/images/papers/ICRC2015-authorlist.pdf
}

The atmospheric depth, $X_{\max }$, at which the particle number of an air shower reaches its maximum is a good indicator for the mass of the primary particle. We present a comparison of the energy evolution of the mean of $X_{\max }$ as measured by the Telescope Array and Pierre Auger Collaborations. After accounting for the different resolutions, acceptances and analysis strategies of the two experiments, the two results are found to be in good agreement within systematic uncertainties.

The 34th International Cosmic Ray Conference,

30 July- 6 August, 2015

The Hague, The Netherlands

${ }^{*}$ Speaker. 

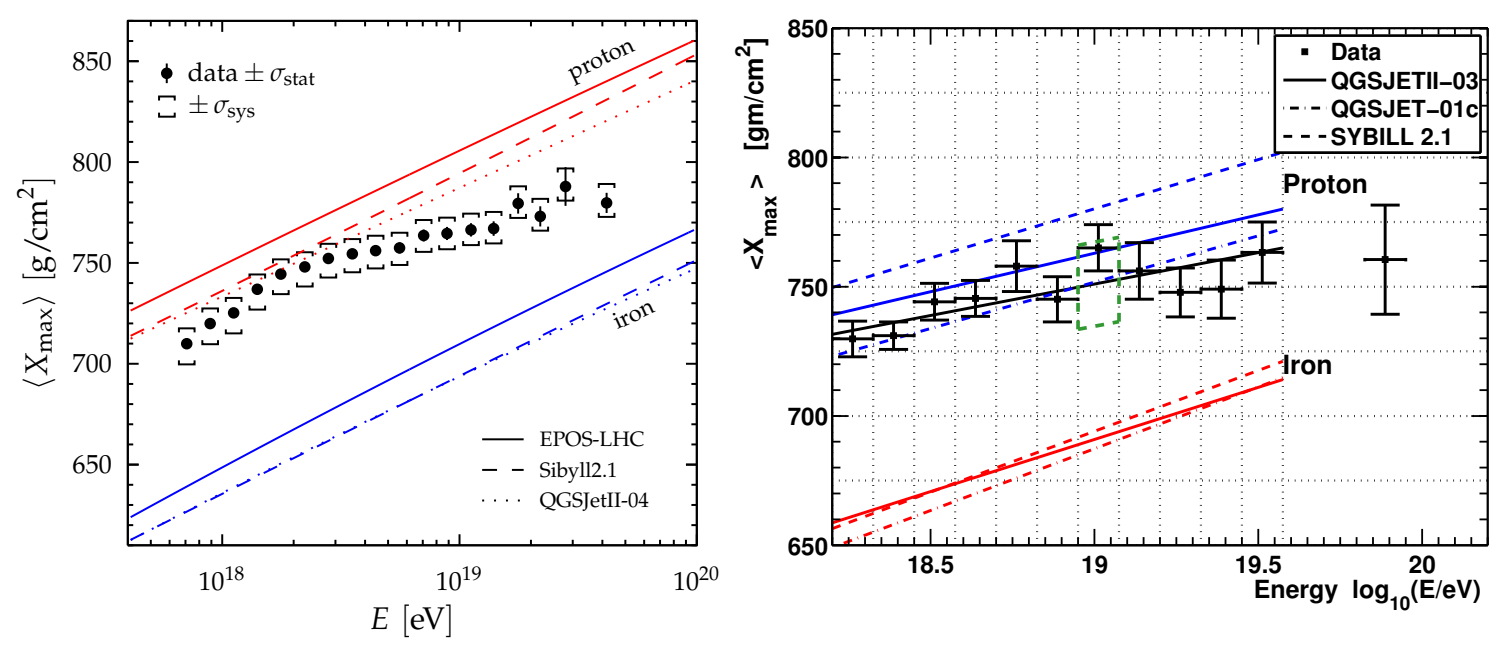

Figure 1: $\left\langle X_{\max }\right\rangle$ as measured by the Pierre Auger (left) and Telescope Array (right) Collaborations [2, 3]. The colored lines denote predictions of air-shower simulations (note that different models are shown in the left and right panel, only SiBYLL2.1 is the same). The black line on the right panel is a straight-line fit to the TA data. Systematic uncertainties are indicated by brackets (left) and by the green dashed box (right).

\section{Introduction}

The nuclear composition of ultra-high energy cosmic rays is one of the key observables to understand their origin. One of the most robust and precise observables to date to infer the composition from air-shower measurements is the atmospheric depth at which the particle number of the shower reaches its maximum, $X_{\max }$. Currently, the Pierre Auger Observatory and the Telescope Array (TA) measure $X_{\max }$ using fluorescence detectors. But despite the use of the same detection principle, a direct comparison of the data published by both collaborations is not straightforward.

The TA Collaboration published values of the average shower maximum, $\left\langle X_{\max }\right\rangle$, obtained from $X_{\max }$ distributions that include detector effects such as the selection efficiency and acceptance. The interpretation of the data is made possible by the comparison of the Monte-Carlo prediction for proton and iron nuclei folded with the same detector resolution and efficiency. In the analysis performed by the Pierre Auger Collaboration, only shower geometries are selected allowing the sampling of almost unbiased $X_{\max }$ distributions and residual biases from the acceptance, reconstruction and resolution are corrected for.

The corresponding values of $\left\langle X_{\max }\right\rangle$ are presented in Fig. 1 together with predictions from air-shower simulations for proton- and iron-initiated showers. SIBYLL2.1, the only hadronic interaction model used by both collaborations, provides a common reference in these plots.

The work reported here is a common effort of the Auger and TA Collaborations with the aim of providing a direct comparison of the $\left\langle X_{\max }\right\rangle$ measurements taking into account the different approaches of each collaboration. Indirect comparisons of TA and Auger results using a conversion of $\left\langle X_{\max }\right\rangle$ to the average logarithmic mass were published in earlier [1]. The disadvantage of indirect comparisons is that they depend on the particular hadronic interaction model that is used. The current analysis was performed in the following way. The Auger $X_{\max }$ distributions were fitted by a combination of four primary nuclei (proton, helium, nitrogen, iron) using events from 
air-shower simulations. The abundances which best fit the Auger data were simulated through the Middle Drum detector of TA (TA-MD) and analyzed by the TA Collaboration using the same procedure as applied to their data. This procedure resulted in the Auger data folded into the TAMD detector. The Auger $\left\langle X_{\max }\right\rangle$ folded with TA-MD analysis is shown in this paper in comparison to the TA-MD data as published [3].

\section{Data Samples}

The analysis presented here is based on the data measured with the Pierre Auger Observatory in the period 1st December 2004 to 31st December 2012. All measured events were analyzed as explained in reference [2]. The events were selected to guarantee good measurement conditions and a high-quality reconstruction. After that, the fiducial selection was applied. In total 19,759 events were considered for further analysis ( 7365 above the lower energy threshold of TA, see below). The $X_{\max }$ values of these events were sampled in 18 energy bins starting at $\log (E / \mathrm{eV})=17.8$.

From the Telescope Array we use hybrid data collected with the MD fluorescence telescope and surface detector array over the period from the 27th May 2008 to 2nd May 2013. The reconstruction and analysis applied to the data are described in [3]. The number of events which passed all cuts is 438 , for which the mean $X_{\max }$ is shown in 12 energy bins above $\log (E / \mathrm{eV})=18.2$.

The number of events used for this comparison presented here is shown in Fig. 2 and the $X_{\max }{ }^{-}$ resolution of the two experiments is presented in Fig. 3. As can be seen, the resolutions after cuts are comparable but it is worthwhile noting that the resolution quoted for the MD does not contain effects from the detector calibration and atmospheric monitoring. The systematic uncertainties on the $X_{\max }$ scale, compared in the right panel of Fig. 3, are $\leq 10 \mathrm{~g} / \mathrm{cm}^{2}$ and $16 \mathrm{~g} / \mathrm{cm}^{2}$ for the Auger and TA analyses respectively.

\section{Analysis}

The relation between the true and observed $X_{\max }$ distribution is

$$
f_{\text {obs }}\left(X_{\max }^{\mathrm{rec}}\right)=\int_{0}^{\infty} f_{\text {true }}\left(X_{\max }\right) \varepsilon\left(X_{\max }\right) R\left(X_{\max }^{\mathrm{rec}}-X_{\max }\right) \mathrm{d} X_{\max },
$$

i.e., the true distribution $f_{\text {true }}$ is deformed by the detection efficiency $\varepsilon$ and smeared by the detector resolution $R$ that relates the true $X_{\max }$ to the reconstructed one, $X_{\max }^{\mathrm{rec}}$.

Due to the different analysis approaches of the TA and Pierre Auger Collaborations it is not possible to compare the published values of the moments of the $X_{\max }$ distribution directly. Whereas $\left\langle X_{\max }\right\rangle$ and $\sigma\left(X_{\max }\right)$ published by the Pierre Auger Collaboration are close to the true moments (i.e. the moments of $f_{\text {true }}$ ), the TA collaboration published the $\left\langle X_{\max }\right\rangle$ folded with the effects of the detector response and reconstruction (i.e. the moments of $f_{\mathrm{obs}}$ ).

To be able to perform a comparison of the two results, we need to establish what $\left\langle X_{\max }\right\rangle_{\text {obs }}$ would be if the $X_{\max }$ distributions measured by Auger were observed by the TA detector. For this purpose, we convolute a parametric description of $f_{\text {true }}$ that is based on the Auger data with the TA detector simulation and apply the same reconstruction and analysis chain used for the TA data to this simulated data set (see [4] for a previous description of this method). 


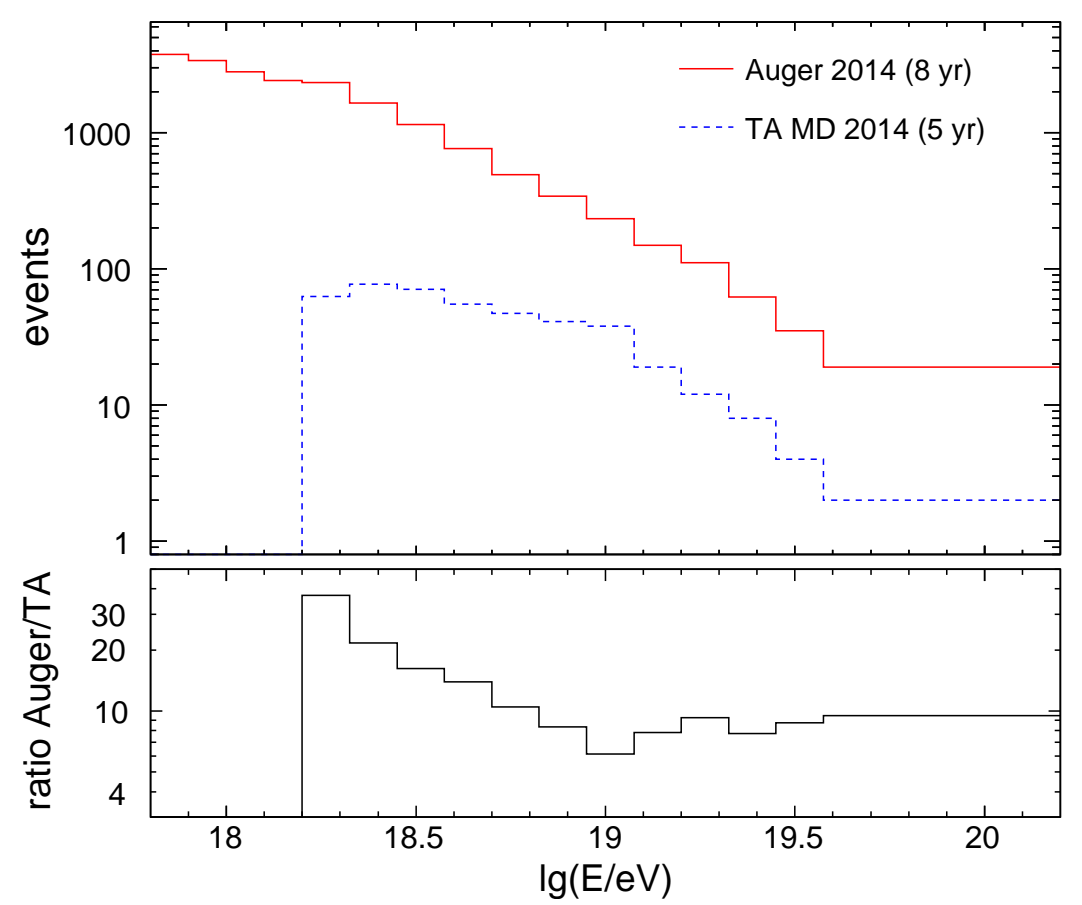

Figure 2: Number of selected events for the Auger (solid red line) and TA (blue dashed line) analyses. The ratio of events is given in the lower panel.
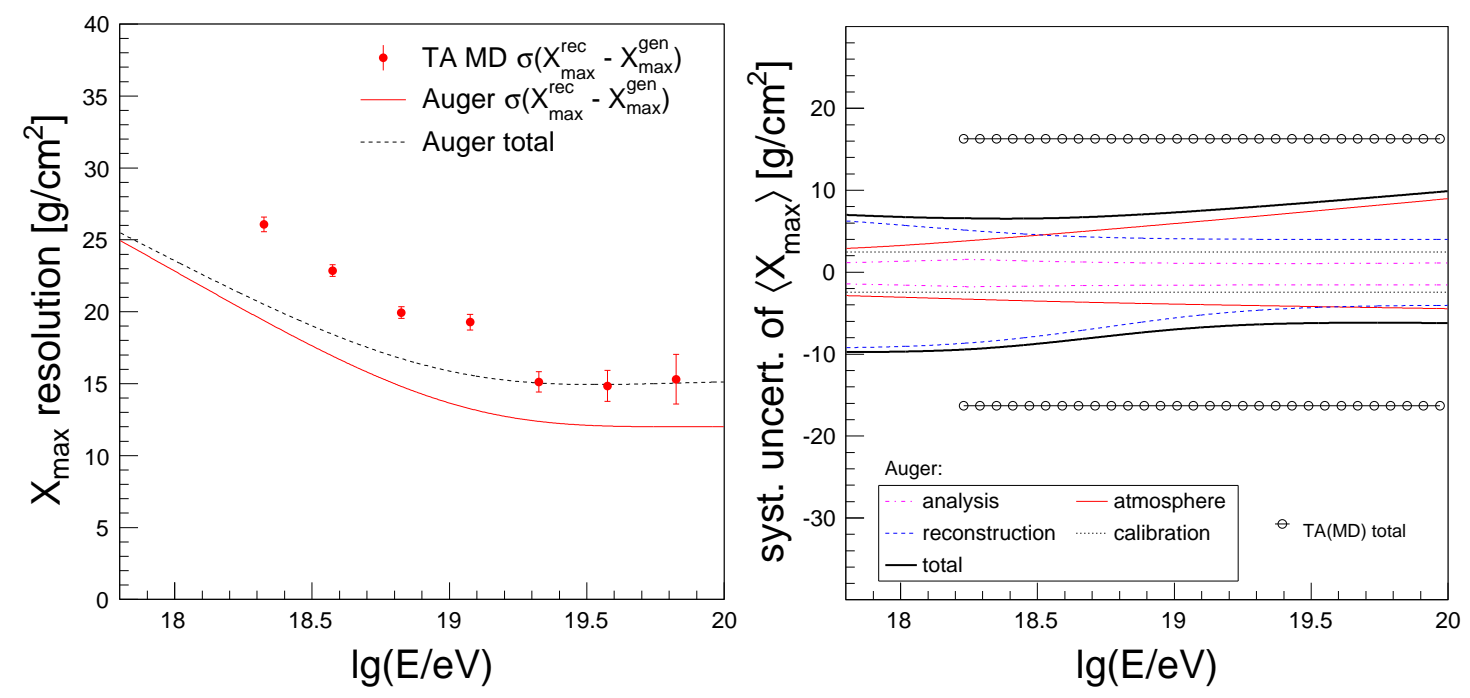

Figure 3: $X_{\max }$ resolution (left) and systematics of the $X_{\max }$ scale (right) for the Auger and TA analyses.

Technically, the parametric description of the $X_{\max }$ distribution is realized by providing a set of composition fractions as a function of energy that describe the $X_{\max }$ distributions measured by Auger. These fractions are obtained as described in [5] by a log-likelihood fit of templates of $X_{\max }$ distributions for different nuclear primaries as predicted by air-shower simulations using a particular hadronic interaction model. It is worthwhile noting that the detector acceptance and resolution at a given primary energy depend mainly on $X_{\max }$ itself and only weakly on the primary 

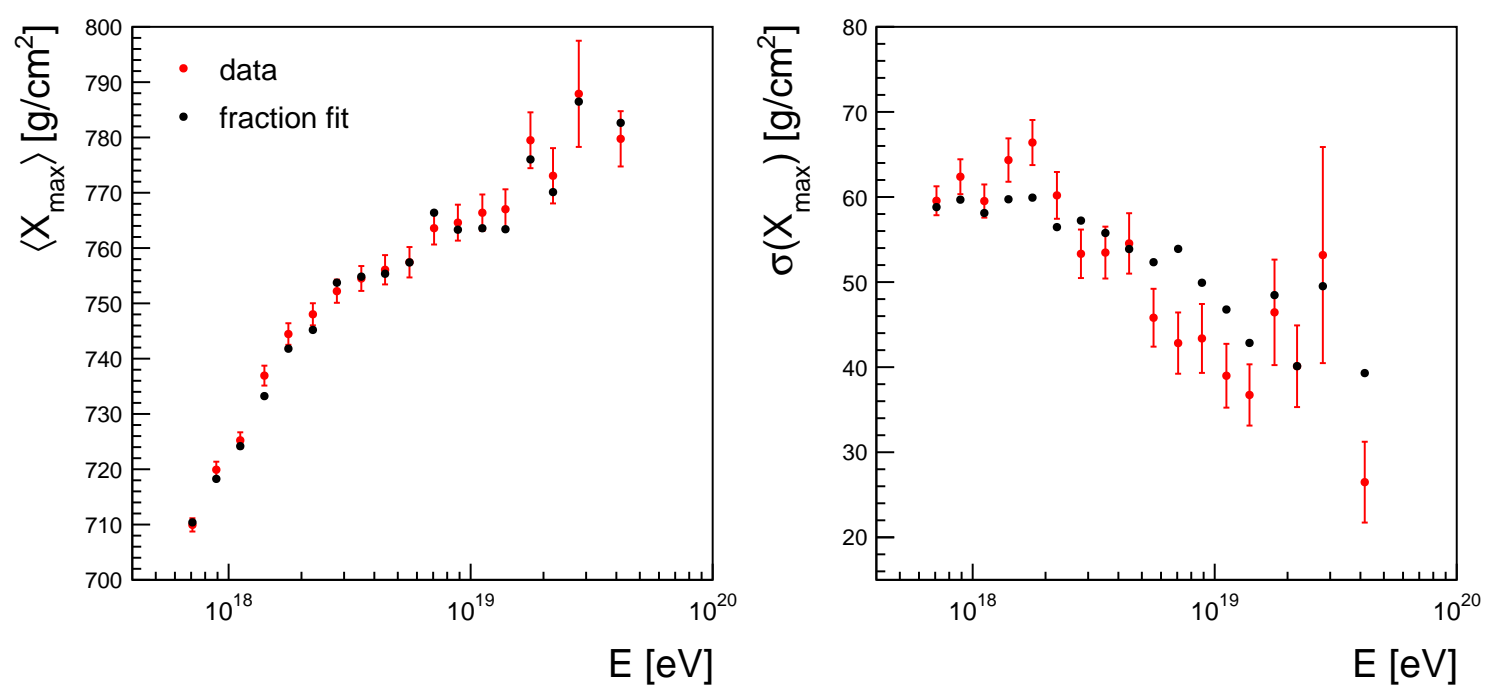

Figure 4: Moments of the fitted $X_{\max }$ distributions using QGSJETII-03 (black markers) and $X_{\max }$ moments measured by the Pierre Auger Collaboration (red circles with statistical error bars), see text.

particle type or hadronic interaction model via the invisible energy. Therefore, for the analysis presented here, it is only important that the resulting composition mix describes the data well and not which fractions of primaries are needed or which hadronic interaction model is used to obtain a model of the undistorted $X_{\max }$ distribution based on Auger data.

Here we used QGSJETII-03 [6] and a mix of four primary particles (proton, helium, nitrogen and iron) to obtain a model of the true $X_{\max }$ distribution based on the Auger data. QGSJETII03 is not included in the set of models studied by the Pierre Auger Collaboration to infer the primary composition [5] because it gives a worse description of LHC data than the re-tuned version QGSJETII-04 [7]. However, with neither version of QGSJETII it is possible to find a composition mix that gives a perfect description of the $X_{\max }$ distributions measured by Auger. The first two moments of the best fits with QGSJETII-03 and the Auger data are shown in Fig. 4. As can be seen, there is a good agreement regarding $\left\langle X_{\max }\right\rangle$, but there are deviations between the fitted and observed width of the distribution.

Ideally, this analysis should be performed with a combination of composition and hadronic interaction model that fits the Auger data well, such as SiB YLL2.1 [8] or EpOS-LHC [9] (see discussion in [5]). However, for practical reasons, we performed a preliminary analysis with QGSJETII03. Since the deviations between the moments of the data and the ones of the fitted distributions are on average at the $5 \mathrm{~g} / \mathrm{cm}^{2}$ level, this approach is expected to give only a small bias in the predictions for the observed distributions.

In detail, the analysis proceeds as follows: the composition mix is processed using the hybridreconstruction-analysis software of the Telescope Array. Showers are generated with CORSIKA and the trigger response of the scintillator array is simulated. The longitudinal shower profile from CORSIKA is fitted to a Gaisser-Hillas function to determine the shower parameters and the fitted profile is used consecutively to generate the light emission. The TA fluorescence detector response including atmospheric, electronics, and geometrical acceptance is then simulated. Subsequently 


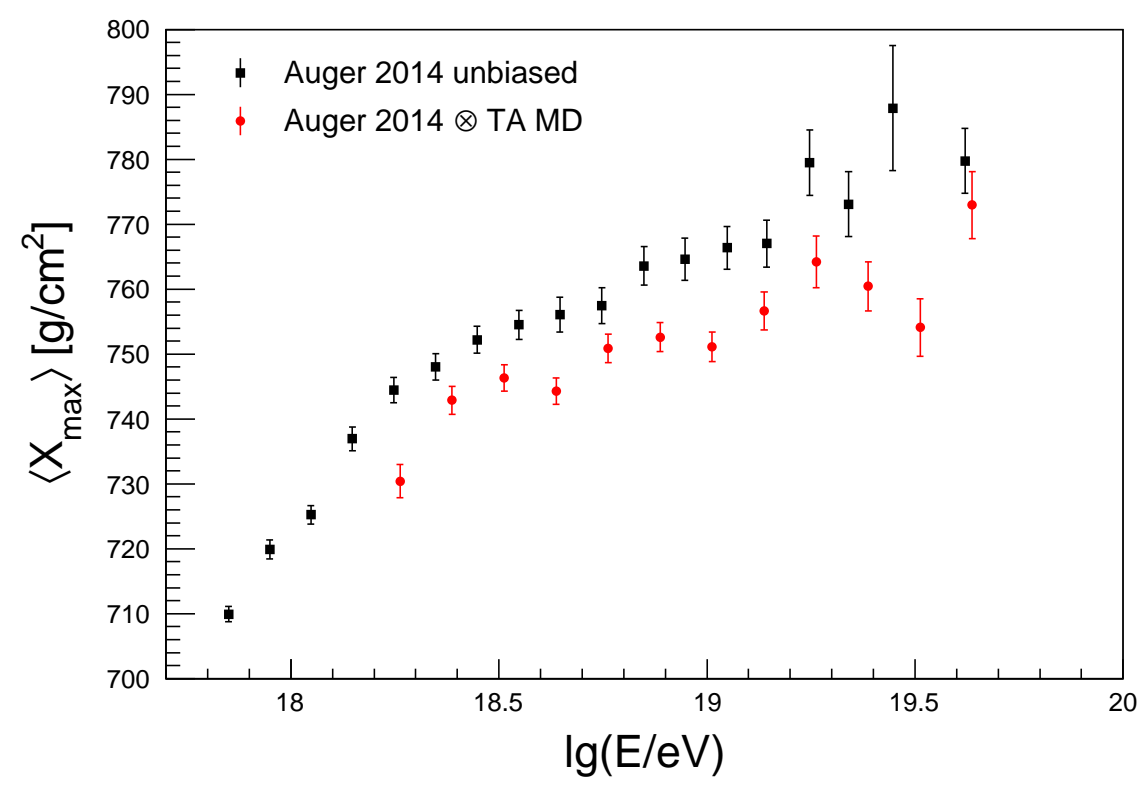

Figure 5: Effect of the MD detector acceptance on $X_{\max }$. The $\left\langle X_{\max }\right\rangle$ of an $X_{\max }$ distribution describing the Auger data before and after the MD acceptance are shown as solid squares and circles respectively. The error bars denote the statistical uncertainties of the Auger result in case of the squares and the statistical uncertainties due to the limited MC statistics in the case of the circles.

the event geometry is fitted via the fluorescence profile, and the shower-detector plane is measured. A fit to hybrid shower geometry is performed which combines the timing and geometric center of charge of the surface detector array, with the timing and geometry of the fluorescence detector that observed the event. This step is what makes the event a "hybrid event". If either the surface or fluorescence detector fail to trigger in an event, it is not processed any further, otherwise the shower profile is fitted via a reverse Monte Carlo method where the atmosphere, electronics, and geometrical acceptance of the shower are fully simulated.

The resulting effect of the folding of the parametric Auger distributions with the TA detector response, reconstruction and analysis on the $\left\langle X_{\max }\right\rangle$ of Auger is shown in Fig. 5. As can be seen, the mean value after the application of the TA detector response is smaller than the generated mean.

\section{Results and Discussion}

The $\left\langle X_{\max }\right\rangle$ as measured by TA using the MD fluorescence telescope and the Auger result folded with the TA acceptance are shown in Fig. 6. Their compatibility is quantified with a binby-bin comparison excluding the highest-energy data points of each experiment which are at different energies. Using only the statistical uncertainties yields a $\chi^{2} /$ Ndf of $10.7 / 11$ with $P\left(\chi^{2} \geq\right.$ $10.7 \mid 11)=0.47$. The average difference of the data points is $(2.9 \pm 2.7$ (stat. $) \pm 18$ (syst. $)) \mathrm{g} / \mathrm{cm}^{2}$ with a $\chi^{2} /$ Ndf of $9.5 / 10(P=0.48)$. It can be concluded that the two data sets are in excellent agreement, even without accounting for the respective systematic uncertainties on the $X_{\max }$ scale. However, in the present study we did not take into account a possible difference in the energy scale of the two experiments. The comparison of the energy spectra at the ankle region suggests that the energy scale of TA is about $13 \%$ higher than the one of the Pierre Auger Observatory [10]. 


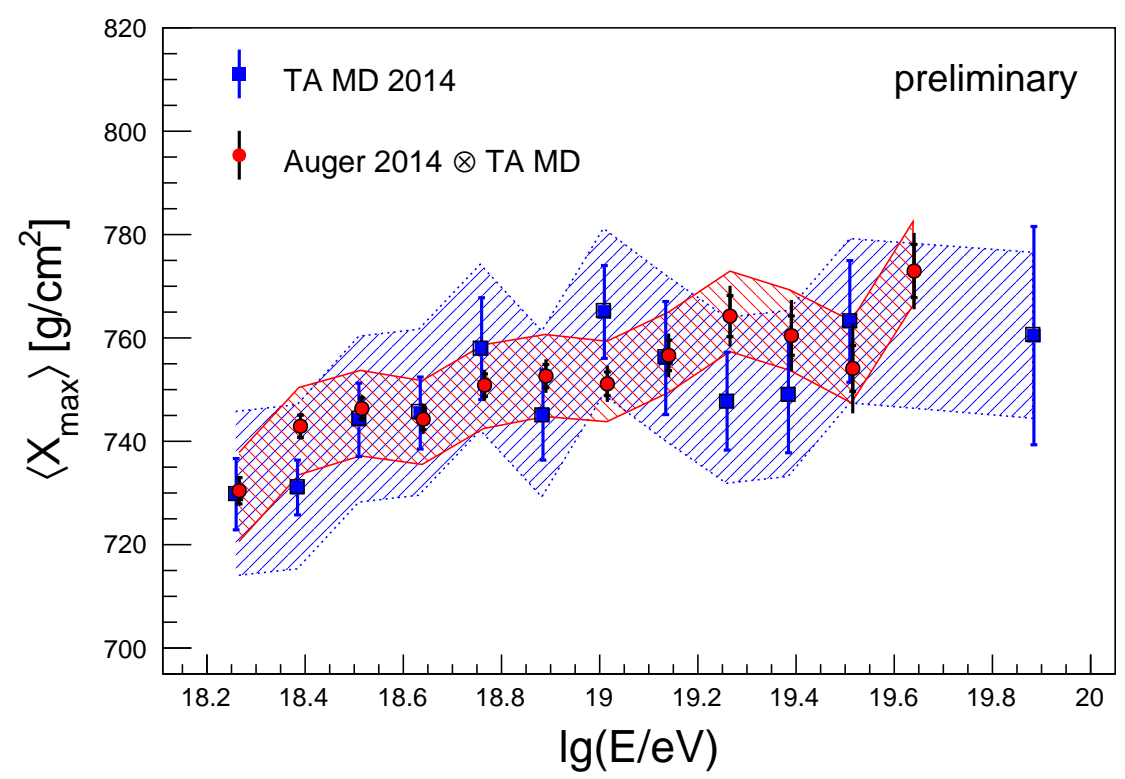

Figure 6: Comparison of $\left\langle X_{\max }\right\rangle$ as measured with the MD of TA (blue squares) and the $\left\langle X_{\max }\right\rangle$ of the Auger data folded with the MD acceptance (red circles). The data points are slightly shifted horizontally for better visibility. In the case of the Auger points, the inner error bars denote the statistical uncertainty of the measurement and the total error bar also includes contributions from the limited statistics of simulated events used for the folding. The colored bands show the systematic uncertainties of the $X_{\max }$ scales of each experiment.

However, since the elongation rate of the folded Auger data is small $\left(\sim 19 \mathrm{~g} / \mathrm{cm}^{2} /\right.$ decade $)$, the effect of such an energy shift on the comparison is expected to be at the level of a few $\mathrm{g} / \mathrm{cm}^{2}$. For a more precise evaluation it would be necessary to take into account the energy dependence of the acceptance of TA. Nevertheless, it is to be expected that the increased difference between the two data sets once the energy scale shift is taken into account will be much smaller than the systematic uncertainties on the $X_{\max }$ scale of $\leq 10 \mathrm{~g} / \mathrm{cm}^{2}$ and $16 \mathrm{~g} / \mathrm{cm}^{2}$ for the Auger and TA analyses respectively.

\section{Conclusions and Outlook}

In this paper we have presented a comparison between the data on $\left\langle X_{\max }\right\rangle$ as measured by the Pierre Auger and Telescope Array Collaborations. An adequate comparison was achieved by taking into account that the $\left\langle X_{\max }\right\rangle$ published by Auger are corrected for detector effects, whereas those published by TA includes detector effects. From the preliminary comparison presented here we conclude that the data of the two observatories are in good agreement.

In the future, we will present results with an improved parametric description of the Auger $X_{\max }$ distributions using the EPOS-LHC interaction model and the evaluation of the effect of the relative energy scale uncertainty. Moreover, we will discuss results from statistical tests of the compatibility of the full $X_{\max }$ distribution. 


\section{References}

[1] E. Barcikowski et al. for the Pierre Auger, Telescope Array and Yakutsk Collaborations, Mass Composition Working Group Report at UHECR-2012, EPJ Web Conf. 53 (2013) 01006

[2] A. Aab et al. [Pierre Auger Collaboration], Depth of maximum of air-shower profiles at the Pierre Auger Observatory. I. Measurements at energies above $10^{17.8}$ eV, Phys. Rev. D 90 (2014) 122005

[3] R.U. Abbasi et al. [Telescope Array Collaboration], Study of Ultra-High Energy Cosmic Ray composition using Telescope Array's Middle Drum detector and surface array in hybrid mode, Astropart. Phys. 64 (2014) 49.

[4] W.F. Hanlon for the Pierre Auger and Telescope Array Collaborations, Progress towards understanding the analyses of mass composition made by the Auger and Telescope Array Collaborations, Proc. 33rd ICRC (2013) [arXiv: 1310 .0647].

[5] A. Aab et al. [Pierre Auger Collaboration], Depth of maximum of air-shower profiles at the Pierre Auger Observatory. II. Composition implications, Phys. Rev. D 90 (2014) 122006.

[6] S. Ostapchenko, Monte Carlo treatment of hadronic interactions in enhanced Pomeron scheme: I. QGSJET-II model, Phys. Rev. D 83 (2011) 014018.

[7] S. Ostapchenko, QGSJET-II: physics, recent improvements, and results for air showers, EPJ Web Conf. 52 (2013) 02001.

[8] E. J. Ahn, R. Engel, T. K. Gaisser, P. Lipari and T. Stanev, Cosmic ray interaction event generator SIBYLL 2.1, Phys. Rev. D 80 (2009) 094003.

[9] T. Pierog, I. Karpenko, J. M. Katzy, E. Yatsenko and K. Werner, EPOS LHC : test of collective hadronization with LHC data (2013) [arXiv: 1306.0121 ].

[10] I. C. Mariş for the Pierre Auger and Telescope Array Collaborations, High Energy Spectrum Working Group Report, presentation at the UHECR symposium (2014), Springdale, USA,

http: //uhecr2014.telescopearray.org/maris/TAAuger_Springdale.pdf 